Session 3: Observations and surveys of massive stars: hot stars, cool stars, transition objects and binaries 


\title{
Highly accurate quantitative spectroscopy of massive stars in the Galaxy
}

\author{
María-Fernanda Nieva and Norbert Przybilla
}

Institut für Astro- und Teilchenphysik, Universität Innsbruck, Technikerstrasse 25/8, 6020 Innsbruck, Austria

email: maria-fernanda.nieva@uibk.ac.at

\begin{abstract}
Achieving high accuracy and precision in stellar parameter and chemical composition determinations is challenging in massive star spectroscopy. On one hand, the target selection for an unbiased sample build-up is complicated by several types of peculiarities that can occur in individual objects. On the other hand, composite spectra are often not recognized as such even at medium-high spectral resolution and typical signal-to-noise ratios, despite multiplicity among massive stars is widespread. In particular, surveys that produce large amounts of automatically reduced data are prone to oversight of details that turn hazardous for the analysis with techniques that have been developed for a set of standard assumptions applicable to a spectrum of a single star. Much larger systematic errors than anticipated may therefore result because of the unrecognized true nature of the investigated objects, or much smaller sample sizes of objects for the analysis than initially planned, if recognized. More factors to be taken care of are the multiple steps from the choice of instrument over the details of the data reduction chain to the choice of modelling code, input data, analysis technique and the selection of the spectral lines to be analyzed. Only when avoiding all the possible pitfalls, a precise and accurate characterization of the stars in terms of fundamental parameters and chemical fingerprints can be achieved that form the basis for further investigations regarding e.g. stellar structure and evolution or the chemical evolution of the Galaxy. The scope of the present work is to provide the massive star and also other astrophysical communities with criteria to evaluate the quality of spectroscopic investigations of massive stars before interpreting them in a broader context. The discussion is guided by our experiences made in the course of over a decade of studies of massive star spectroscopy ranging from the simplest single objects to multiple systems.
\end{abstract}

Keywords. stars: parameters, stars: abundances

\section{Introduction}

We focus our discussion on stars with masses between $\sim 20$ and $\sim 6$ solar masses. From the spectral characteristics, this corresponds to the latest $\mathrm{O}$ stars to early B stars on the Main Sequence, the most numerous but also the simplest massive stars to be studied. In contrast to earlier spectral types, supergiants or Be stars, they are not significantly affected by mass outflow or complex geometries. And, in comparison to many mid/late B-type stars, they do not present signatures of atomic diffusion in their atmospheres. Many model atmospheres and analysis methods for massive star spectra have therefore been optimized to study these simple objects. The typical assumptions are that they are single, they have stationary, hydrostatic and chemically homogeneous atmospheres, are described well by plane-parallel geometry and (non-)local thermodynamic equilibrium.

However, in order to address the topic of massive stars in its entirety, one has to analyze earlier O stars as well, OB supergiants, Be and late B-type stars, chemically peculiar, 
magnetic, pulsating, fast-rotating stars, and also pre-main-sequence stars. The large variety of massive stars challenges the basic assumptions of standard atmospheric models and spectral analysis techniques that have been often used to estimate their fundamental parameters and chemical composition. One has to consider e.g. (inhomogeneous) hydrodynamic outflows, oblateness of the stars because of high rotational velocities and the presence of an accretion/decretion disk surrounding them (i.e. non-spherical geometries), the effects of spots on stellar surfaces due strong magnetic fields, elemental abundance stratification due to diffusion, deformation of spectral lines due to stellar pulsations, etc. Practically all these topics have been addressed by dedicated studies. However, a unique tool to (automatically) analyze spectra formed under such a variety of conditions is not implemented currently. The limiting factor is the large number of physical assumptions to be made for the general case, preventing an efficient calculation of models covering the entire parameter space.

A more feasible approach to reliably study each kind of star is via tailored model atmospheres and analysis techniques that incorporates the most realistic physical background for each particular case. Dedicated spectral grids can then be computed for each kind of object when the range in all relevant parameters is known. The final scope is to derive the stars' atmospheric parameters to reliably place them in a Kiel diagram (surface gravity $\log g$ vs. effective temperature $\left.T_{\text {eff }}\right)$. In cases where fundamental parameters can also be derived (with additional information, like e.g. parallaxes), the objects can be placed in a Hertzsprung-Russell diagram, and basic relationships between fundamental stellar parameters can be checked. Related to the atmospheric and fundamental parameters is the determination of the stars' spectroscopic distances, that - when compared with other independent distance indicators - allow us to constrain the accuracy of the stellar parameters. Spectral energy distributions from the UV to the IR allows us to derive the extinction and reddening for a particular star, and to cross-check for it's global energy output.

A subsequent step after the stellar parameter determination is the chemical analysis of trace elements, which also differs among the various kind of stars because of e.g., temperature constraints, where lines of different species/ionization stages vary in their strength, different blends appear in different parts of the spectrum, or some lines can turn from absorption to emission. The projected rotational velocity will also determine which lines can be analyzed in isolation and which blends can be consistently taken into account. A detailed chemical analysis can yield on accurate elemental abundances when four basic conditions are met: i) the stellar atmospheric models are applicable to the objects, ii) the spectra have a good quality (spectral lines should be resolved and measurable at good $\mathrm{S} / \mathrm{N}$ ), iii) the analysis methodology takes into account a good selection of spectral lines that can be well reproduced by the model spectra and iv) the atmospheric (and when possible fundamental) parameters have been consistently derived.

This manuscript is biased towards our experience on the spectral modeling and analysis of normal and peculiar massive single and recently also multiple stars. It discusses some successes but also challenges to current modeling capabilities. It is intended as a guideline for colleagues working in other areas to assess the accuracy and precision of published fundamental parameters and chemical abundances for massive stars, before they are interpreted in various astrophysical contexts. A special emphasis is put on the single OB stars on the Main Sequence, because they allow us to derive most parameters and chemical abundances at highest accuracy and precision, and therefore, we can consider them as reference objects. Shorter descriptions are dedicated to particular classes of more complex objects for which we have recently extended our spectral analyses. 


\section{Late-O and early B-type on the Main Sequence: the simplest stars}

For over a decade, we have improved the spectral modeling and analysis technique for stars with spectral classes from O9 to B2 and luminosity classes V to III - the socalled OB subgroup because of their similar spectral characteristics. Given that standard stellar model atmospheres like e.g. Atras9 (Kurucz 1993) meet most requirements to reproduce their atmospheric structures well (Nieva \& Przybilla 2007), our efforts were invested into realistic level population and line-formation computations in non-LTE by building and testing different configurations of new input atomic data to provide with robust model atoms for different elements (see Przybilla et al. 2016 for updated references). The codes used for the non-LTE level population and line-formation calculations are Detail (Giddings 1981) and Surface (Butler \& Giddings 1985)†. Our new spectral modeling in combination with a self-consistent spectral analysis that accounts for multiple ionization equilibria, applied to high-quality observations, resulted in a drastic minimization of stellar parameter and chemical abundance uncertainties, particularly reducing several systematic effects previously unaccounted for. A comprehensive study and first applications of our work on single and normal early B-type dwarfs and giants were discussed by Nieva \& Przybilla (2012, 2014, hereafter NP12 and NP14) and Nieva (2013). These studies allowed us to put constraints not only on their atmospheric parameters and chemical composition at high precision and accuracy, but also to derive spectroscopically other stellar parameters like radius, luminosity, mass and to explore whether theoretical relations between them hold using our observationally derived parameters. For such stars, uncertainties in effective temperature, surface gravities and chemical abundances as low as 1-2\%, $15 \%$ and $25 \%$, respectively (NP12) and in evolutionary masses, radii and luminosities better than 5\%,10\%,20\%, respectively and in absolute visual and bolometric magnitudes lower than $0.20 \mathrm{mag}$ (NP14) are achieved. Moreover, practically a perfect match between the observed and the computed spectrum per star for one set of parameters confirm the robustness of models and analysis. Figure 1 shows a precise location of a sample of single early B-type stars (NP12) in the Kiel diagram in comparison to detached eclipsing binaries (DEBs). Figure 2 shows their mass-radius relation and Fig. 3 their mass-luminosity relation (NP14), indicating that the derived stellar parameters lie within theoretically expected values and agree with more accurate results from DEBs. The empirical relation between absolute magnitudes and spectral types in NP14 show an offset with respect to classical older calibrations from the literature (Cox 2000). The latter are often adopted, affecting the computation of e.g. the stellar luminosities. The level of accuracy reached for this kind of objects can hardly be reproduced for more complex stars or systems. We therefore use these results as references for further studies.

\section{BA and OB supergiants}

Supergiants of spectral types late-B and early-A are descendants of OB stars on the Main Sequence. Their atmospheres can be well modeled with AtLas9, Detail and SurFACE, as described in Przybilla et al. (2006). These objects also have the advantage that they outshine practically any companion in the visual, facilitating to treat them as single stars. The spectral analysis of early-O stars and OB supergiants is beyond the modeling capability of this hydrostatic approach, however other robust hydrodynamic non-LTE codes that treat simultaneously the spherically symmetric stellar atmospheric structure

$\dagger$ For this type of stars, LTE atmospheric structures computed with ATLAS9 and stellar fluxes computed with AtLas9 and Detail are practically identical to those calculated with TLusty Hubeny \& Lanz (1995) in non-LTE, see Nieva \& Przybilla (2007) for a detailed discussion. 


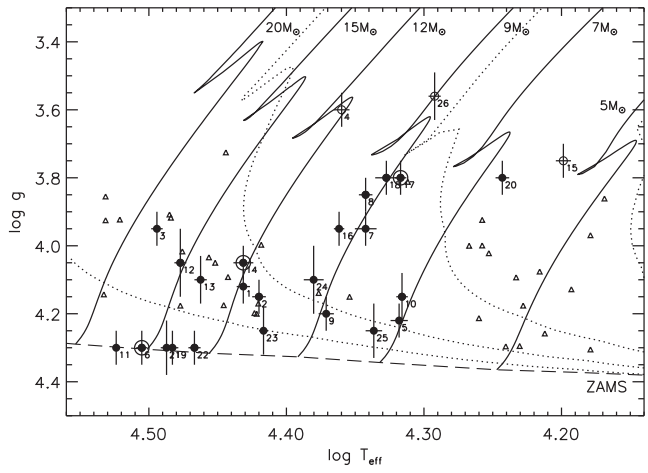

Figure 1. $T_{\text {eff }}$ and $\log g$ of a sample of early B-type stars on the Main Sequence (black dots). Open thick circles are objects beyond core H-exhaustion. Wide circles surrounding the dots mark magnetic stars. Data from double-lined detached eclipsing binaries are shown as small triangles. Evolutionary tracks and isochrones from Ekström et al. (2012) are shown. See NP12 and NP14 for details.

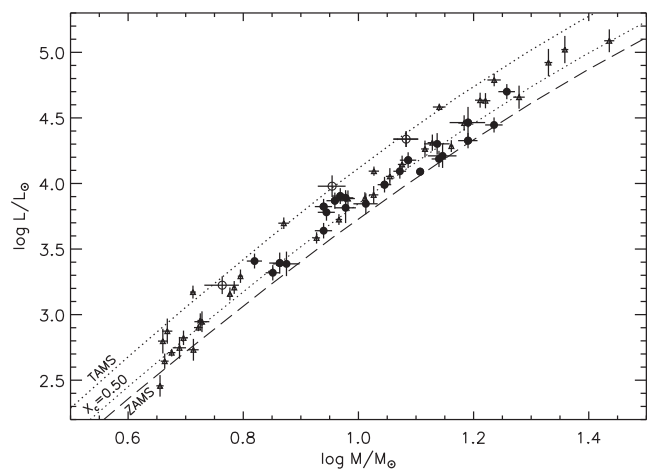

Figure 3. Mass-luminosity relationship for the sample stars in Fig. 1. Symbol and loci encoding are the same as in Figs. 1 and 2 (NP14).

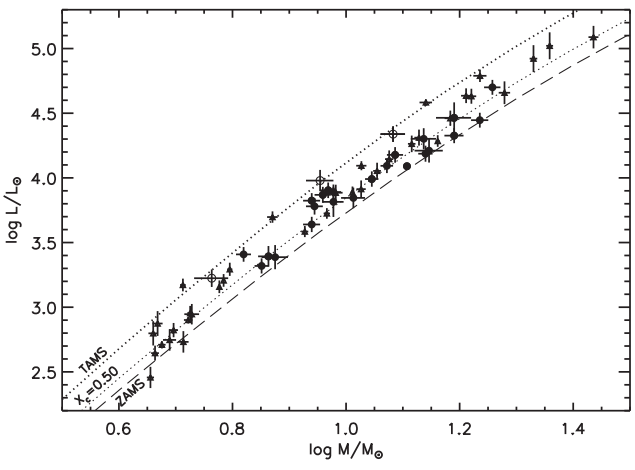

Figure 2. Mass-radius relationship for the sample stars in Fig. 1 with the same symbol encoding. Abscissa values are evolutionary masses. The ZAMS and two additional loci for $50 \%$ core- $\mathrm{H}$ depletion and for the TAMS are indicated by the thick/thin-dotted lines from the stellar evolution code as in Fig. 1. Error bars are shown also for the detached eclipsing binaries. See NP14 for details.

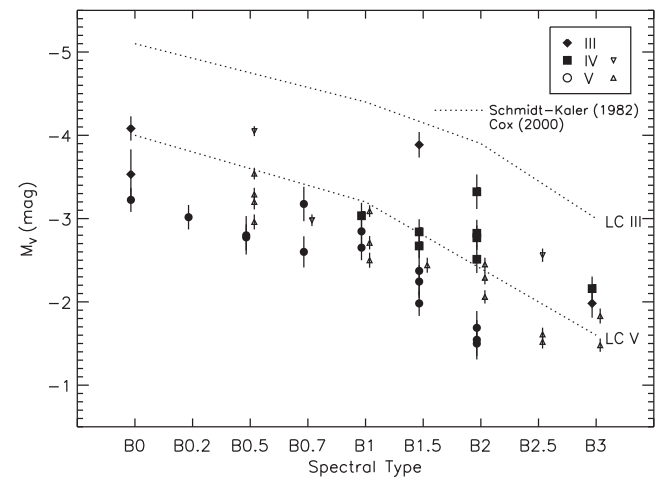

Figure 4. Absolute visual magnitudes of the sample stars in Fig. 1, encoding their luminosity class according to the legend, vs. spectral type. Older reference values are also indicated (NP14).

and the stellar wind like CMFGen (Hillier \& Miller 1998) and FAstwind (Puls et al. 2005) are suited for their study.

\section{Fast-rotating stars}

We encounter several limitations in the analysis of intrinsically fast-rotating stars. If the star rotates so fast that its shape is affected (oblate), the assumptions of plane-parallel or even spherical symmetry are no longer valid on the global scale. Also the temperature will vary between the poles and the equator. Depending on the star's inclination, we observe spectral lines formed at different atmospheric conditions, with different local temperatures. This is clearly evident in cases of fast-rotators seen pole-on, where the lines are sharp, but nevertheless it is difficult to fit the whole spectrum with one temperature only and the assumption of establishing ionization balance for all available 
species simultaneously is no longer valid. If the projected rotational velocity is large, only a few spectral lines can be analyzed and many blends have to be accounted for self-consistently. If the $\mathrm{S} / \mathrm{N}$ ratio is not high enough, the definition of the line continuum is one of the largest sources of systematics because the spectral lines get smeared out and appear weaker (see Korn et al. 2005 for a discussion). The analysis of fast-rotating stars is challenging and the limitations have to be established on a case-by-case basis. One of the most difficult tasks, when the stars have large projected rotational velocities, is identifying systematic asymmetries that can be caused by a companion in a spectroscopic binary (or multiple) system. Stellar parameters and chemical abundances derived from these kind of objects should be treated extremely carefully, because they are prone to large systematic errors. Maeder et al. (2014) provide in their Table 1 an example of a re-assessment of a sub-sample of objects from the Flames Massive Star Survey studied by Hunter et al. (2009), resulting in the identification of stars with previously unnoticed double or asymmetric spectral lines, which cause their natural exclusion from the interpretation of results because they were analyzed with standard techniques.

\section{Be stars}

Be stars are main-sequence or subgiant stars of spectral type B characterized by Balmer emission (e.g., $\mathrm{H} \alpha$, but also other Balmer and eventually metal lines) that originates in a circumstellar disk. As most Be-stars are fast-rotators, the same limitations exposed in Sect. 4 apply to their analysis. Additionally, the clear signatures of lines formed in the disk cannot be reproduced self-consistently with any stellar atmosphere code at present. However, there are cases where the photospheric lines can be successfully reproduced, constraining reliably the stellar parameters and even metal abundances. An exploration of the extent of applicability of our spectral models and methods to such stars is ongoing.

\section{Mid- and late B-type stars}

Mid- and late B-type stars present fewer metal lines and less elements with different ionization stages traced in the spectra ( 1 or 2 ), in contrast to hotter stars (4 to 6$)$. In many cases, in addition, the stars have a large projected rotational velocity, which can turn their analysis even more challenging. The identification of a companion forming a spectroscopic binary system, by resolving line-asymmetries is also a challenge for many of these stars (Zwintz et al., subm.). Many objects show chemical peculiarities due to atmospheric diffusion, others present normal chemical abundances.

\section{Chemically peculiar, pulsating and magnetic stars}

Chemically peculiar stars are stars with distinctly unusual metal abundances in their surface layers. The stars present selective diffusion of different elements in their atmospheres causing some elements to show higher or lower abundances in their outer layers. Helium-strong stars constitute the hottest and most massive chemically peculiar stars of the upper Main Sequence. The chemical abundances and metallicities of such stars should be accounted for iteratively in every step of the analysis by computing dedicated model spectra, because their peculiar abundances are not considered in pre-computed grids of model spectra. An example of the consequence of not taking the peculiar abundances into account in the stellar parameter and chemical composition determination, in contrast to a self-consistent analysis is shown in Figs. 5 and 6, adapted from Przybilla et al. (2016). Analyses assuming solar helium abundances, in contrast to the enhanced 


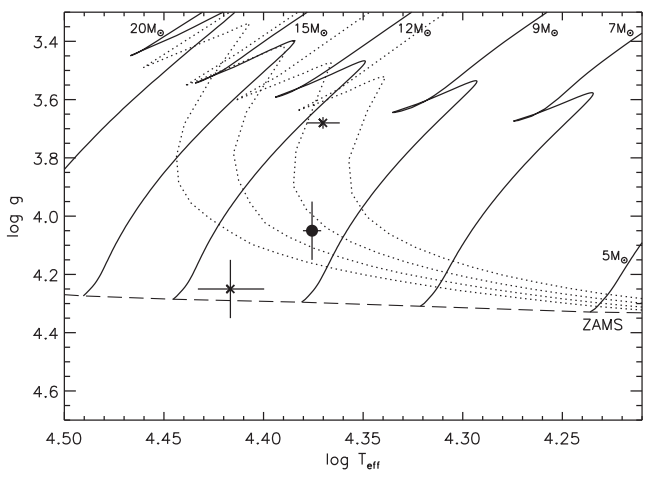

Figure 5. CPD-57 3509 in the Kiel diagram. Black dot: result from our self-consistent analysis. St. Andrew's crosses: results discussed in the literature at higher and lower lower gravity assuming solar helium abundance. Adapted from Przybilla et al. (2016).

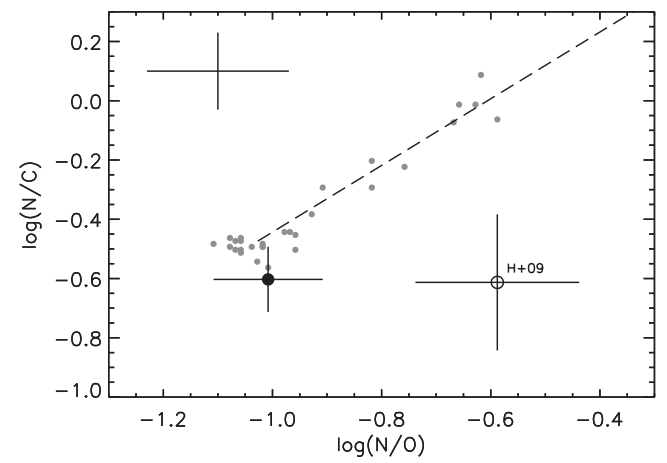

Figure 6. CPD-57 3509 in the N/O-N/C diagram (by mass). Black dot: Przybilla et al. (2016). Open circle: Hunter et al. (2009). Grey dots: early B-type stars from NP12. Dashed line: analytical approximation to the nuclear path for $\mathrm{CN}$-cycled material using initial values from NP12.

helium abundance present in the star, can result in different atmospheric parameters, as seen in Fig. 5 and consequently in different chemical abundances, as shown in Fig. 6 . The same methodology used by Przybilla et al. (2016) was consequently applied to other He-strong stars by Gonzalez et al. (2017), Castro et al. (2017), Briquet et al. (in prep.).

Other less-massive chemically peculiar stars (mid- to late B-type) like He-weak or HgMn stars may pose an extra challenge in their spectral analysis caused by elemental abundance stratification in their atmospheres. Clear signatures of He abundance stratification are noticed e.g. in $\kappa$ Cancri, even when the modeled spectra are computed in non-LTE, whereas oxygen seems not to be stratified in non-LTE, therefore the ionization balance using lines of $\mathrm{O}$ I and $\mathrm{O}$ II formed in different depths in the atmosphere is met when considering non-LTE line formation Maza et al. (2014).

Strongly-pulsating stars present spectral lines with noticeable asymmetries. As model atmosphere codes usually do not include modes of stellar pulsations in their computations, such asymmetries cannot be reproduced typically, but see Irrgang et al. (2016). We notice, however, that some spectral lines are more symmetric because they are formed in regions where the pulsations affect the atmosphere less. In a case-by-case study, it is possible to derive atmospheric parameters through the analysis of the most symmetric lines. An example can be found in Briquet et al. (2011). Note, however, the difference in $\log g$ resulting from the spectroscopic and from the asteroseismic analysis in that case, which should be further investigated. We were also able to reproduce the spectra of several magnetic stars, with magnetic field strengths ranging from very weak to very strong. Some examples are discussed in Fossati et al. (2015), Przybilla et al. (2016), Gonzalez et al. (2017), Castro et al. (2017), Briquet et al. (in prep.), within the "B fields in OB stars" (BOB) collaboration.

\section{Multiple systems}

The spectral line fitting of the triple system HD 164492C in the Trifid Nebula, which is formed by a close spectroscopic binary composed by a normal early B-type star and a chemically peculiar late B-type star and a tertiary He-strong star, has been successfully realized by us in Gonzalez et al. (2017). The number of parameters involved in the spectral fitting is much larger than in the case of single stars. The challenge of the analysis 


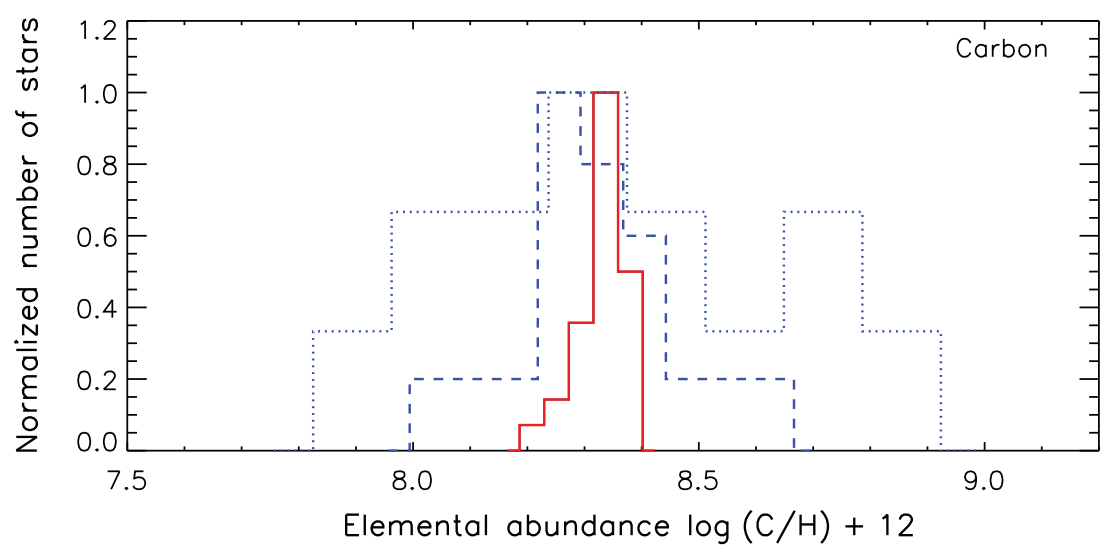

Figure 7. Present-day carbon abundances derived from early B-type stars in the Galaxy. Blue dashed line: apparently normal single stars. Blue dotted line: previously unnoticed SB2 candidates. The stars are distributed in the Galactic disc covering 4 to $15 \mathrm{kpc}$ Galactocentric distance. For comparison, in red full line are carbon abundances of normal single early B-type stars in the solar neighborhood as derived by NP12.

consists in the constraint that the spectroscopic solution should provide consistent ages, spectroscopic distances, mass ratio of the primary system and flux scaling factors. And additionally, because of the chemically peculiar composition of the He-strong star, dedicated grids of model spectra have to be computed to derive parameter combinations for $T_{\text {eff }}, \log g$, microturbulence, projected rotational velocity, macroturbulence, helium abundance and individual metal abundances per star. Within the whole procedure, several parameters are interrelated with each other, therefore the analysis is intensively iterative. In the particular case of HD $164492 \mathrm{C}$, the Balmer lines do not allow to distinguish the contribution of the two brighter components, but the He I lines and the stronger Si III lines show the contribution of the faster-rotator He-strong star on the spectral line wings. The parameters of the faintest star (with a flux contribution of about 4-5\%) cannot be determined from the composite spectrum. Instead, the disentangled spectrum can provide us with some constraints on stellar parameters. This kind of analysis is feasible to be done when time series spectra are available, which is time-consuming for faint objects.

\section{Present-day carbon abundances in the Galactic disk as an application of accurate quantitative spectroscopy in the Galaxy}

We have analyzed new high-resolution spectra taken with UVES@VLT of early Btype stars distributed along the Galactic disk covering Galactocentric distances ranging from 4 to $15 \mathrm{kpc}$. The sample was selected from the literature, and the high-quality data allowed us to identify new SB2 candidates. Preliminary carbon abundances are presented in Fig. 7. The whole sample has been analyzed assuming them as single stars, as it was done in previous work. However, if we separate stars with previously unnoticed SB2 signatures (blue dotted line, where the chosen analysis approach is inappropriate), the carbon abundance distribution is much broader than the sample of apparently single stars (blue dashed line), which still should present an intrinsic spread due to effects of the Galactic abundance gradient. Carbon abundances of apparently single early B-type stars in the solar neighborhood (red full line) as derived in NP12, are also plotted for comparison. 
This is a clear case where - besides improvements in the spectral modeling and analysis technique - the data quality (spectral resolution, $\mathrm{S} / \mathrm{N}$ ratio and wavelength coverage) is decisive for avoiding systematic errors. In this case, the source of systematics lies in the previously unrecognized composite nature of some spectra, which need to be excluded from the analysis based on standard assumptions.

\section{Acknowledgements}

MFN acknowledges support by the Austrian Science Fund through the Lise Meitner program N-1868-NBL.

\section{References}

Briquet, M., et al. 2011, A\& A, 527, A112

Butler, K. \& Giddings, J. R. 1981, Newsletter on Analysis of Astronomical Spectra, no. 9, University College of London

Castro, N., et al. 2017, A\&A, 597, L6

Cox, A. N. 2000, Allen's astrophysical quantities, New York, AIP Press, Springer

Ekström, S., et al. 2012, A\&GA, 537, A146

Fossati, L., et al. 2015, A\&SA, 574, A20

Giddings, J. R. 1981, Ph.D. thesis, University of London

Gonzalez, J. F., et al. 2017, MNRAS, 467, 437

Hillier, D. J. \& Miller, D. L. 1998, ApJ, 496, 407

Hubeny, I. \& Lanz, T. 1995, ApJ, 439, 875

Hunter, I., et al. 2009, A\&SA, 496, 841

Irrgang, A., et al. 2016, A\&BA, 591, L6

Korn, A. J., Nieva, M. F., Daflon, S., \& Cunha, K., 2005, ApJ, 633, 899

Kurucz, R. 1993, CD-ROM No. 13, Cambridge, Mass.: Smithsonian Astrophysical Observatory Maeder, A., et al. 2014, A\&A, 565, A39

Maza, N. L., Nieva, M.-F., \& Przybilla, N. 2014, A\&A A, 572, A112

McSwain, M. V., Huang, W., \& Gies, D. R. 2009, ApJ, 700, 1216

Nieva, M.-F. 2013, A\&\&A, 550, A26

Nieva, M. F. \& Przybilla, N. 2007, A\&A, 467, 295

Nieva, M.-F. \& Przybilla, N. 2012, A\&A, 539, A143

Nieva, M.-F. \& Przybilla, N. 2014, A\& A, 566, A7

Przybilla, N., Butler, K., Becker, S. R., \& Kudritzki, R. P. 2006, A\&\&A, 445, 1099

Przybilla, N., et al. 2010, A\&SA, 517, A38

Przybilla, N., et al. 2016, A\&A, 587, A7

Puls, J., et al. 2005, A\&A A, 435, 669 